\title{
BMJ Open Estimating the prevalence of heterozygous familial hypercholesterolaemia: a systematic review and meta-analysis
}

\author{
Leo E Akioyamen, ${ }^{1,2}$ Jacques Genest, ${ }^{3,4}$ Shubham D Shan, ${ }^{1,2}$ Rachel L Reel, ${ }^{1}$ \\ Jordan M Albaum, ${ }^{1}$ Anna Chu, ${ }^{1,2}$ Jack V Tu ${ }^{1,2,5}$
}

To cite: Akioyamen LE, Genest J, Shan SD, et al. Estimating the prevalence of heterozygous familial hypercholesterolaemia: a systematic review and meta-analysis. BMJ Open 2017;7:e016461. doi:10.1136/ bmjopen-2017-016461

- Prepublication history and additional material are available. To view please visit the journal online (http://dx.doi.org/10. 1136/bmjopen-2017-016461).

Received 16 February 2017 Revised 17 June 2017 Accepted 5 July 2017

CrossMark

${ }^{1}$ Faculty of Medicine, University of Toronto, Toronto, Canada ${ }^{2}$ Institute for Clinical Evaluative Sciences, Toronto, Canada ${ }^{3}$ Faculty of Medicine, McGill University, Montreal, Canada ${ }^{4}$ MGill University Health Centre, Royal Victoria Hospital, Montreal, Canada

${ }^{5}$ Schulich Heart Centre, Sunnybrook Health Sciences Centre, Toronto, Canada

Correspondence to Dr Jack V Tu; tu@ices.on.ca

\section{ABSTRACT}

Objectives Heterozygous familial hypercholesterolaemia (FH) confers a significant risk for premature cardiovascular disease (CVD). However, the estimated prevalence of $\mathrm{FH}$ varies substantially among studies. We aimed to provide a summary estimate of $\mathrm{FH}$ prevalence in the general population and assess variations in frequency across different sociodemographic characteristics.

Setting, participants and outcome measures We searched MEDLINE, EMBASE, Global Health, the Cochrane Library, PsycINFO and PubMed for peer-reviewed literature using validated strategies. Results were limited to studies published in English between January 1990 and January 2017. Studies were eligible if they determined FH prevalence using clinical criteria or DNA-based analyses. We determined a pooled point prevalence of $\mathrm{FH}$ in adults and children and assessed the variation of the pooled frequency by age, sex, geographical location, diagnostic method, study quality and year of publication. Estimates were pooled using random-effects metaanalysis. Differences by study-level characteristics were investigated through subgroups, meta-regression and sensitivity analyses.

Results The pooled prevalence of FH from 19 studies including 2458456 unique individuals was $0.40 \%$ (95\% $\mathrm{Cl} 0.29 \%$ to $0.52 \%$ ) which corresponds to a frequency of 1 in 250 individuals. FH prevalence was found to vary by age and geographical location but not by any other covariates. Results were consistent in sensitivity analyses.

Conclusions Our systematic review suggests that FH is a common disorder, affecting 1 in 250 individuals. These findings underscore the need for early detection and management to decrease CVD risk.

\section{BACKGROUND}

The frequency of heterozygous familial hypercholesterolaemia $(\mathrm{FH})$ was originally reported as 1 in $500(0.2 \%) .{ }^{1}$ This estimate is based on work that determined the prevalence in homozygous individuals and used Hardy-Weinberg principles to calculate the frequency in heterozygotes. ${ }^{2}$ Similar frequencies have been described in subsequent reports of population-based samples. ${ }^{3-7}$
Strengths and limitations of this study

- Use of an extensive search strategy and adherence to predetermined inclusion/exclusion criteria.

- Use of evidence-based inverse variance weighted random effects meta-analysis to quantify a robust estimate of the pooled frequency of heterozygous familial hypercholesterolaemia in adults.

- Our study possesses a large sample size $(n=2458456)$.

- We include only English-language peer-reviewed studies making it possible that some relevant articles were not included.

- Our analyses possessed considerable amount of quantifiable heterogeneity.

However, this estimate has recently been criticised for its imprecision. ${ }^{8}$ Human behaviour does not adhere to Hardy-Weinberg assumptions (eg, random mating, no migration) and violations of these principles have been shown to significantly impact the results of gene-disease association studies. ${ }^{9}$ Further, recent work indicates as many as 1 in 200 people may be affected by $\mathrm{FH}^{10-12}$ and there are some data to suggest that regional variations in FH frequency exist. ${ }^{13-19}$

The population prevalence of $\mathrm{FH}$ is difficult to determine for several reasons. Most countries lack national $\mathrm{FH}$ registers or large observational databases. Yet, even when such databases exist, they often contain insufficient data on aspects of clinical histories essential for $\mathrm{FH}$ diagnosis. No uniform criteria for $\mathrm{FH}$ diagnosis exist and the three sets of criteria commonly used vary in the amount of emphasis placed on clinical characteristics in determining FH. Additionally, the ability to detect such findings may vary based on the clinical acumen and experiences of assessors. ${ }^{20}$ Genetic diagnosis has the potential to mitigate confounding inherent 
in clinical diagnostic criteria. However, the feasibility and cost-effectiveness of genetic screening continues to be debated, ${ }^{81-23}$ a high proportion of patients with clinical FH diagnoses may not be identified ${ }^{24}$ and all of the genetic mutations that cause FH may not yet be known. Together, these factors suggest the potential for a different FH frequency than original estimates.

Ascertaining the prevalence of $\mathrm{FH}$ has important clinical and public health implications, especially in light of the availability of new but expensive treatments (eg, proprotein convertase subtilisin/kexin type 9 (PCKS9) inhibitors) for this condition. FH is caused by defects in the low-density lipoprotein receptor (LDLR) pathway, resulting in elevated LDL-cholesterol (LDL-C) concentrations that are largely resistant to caloric restriction, weight loss and physical exercise interventions in affected individuals. ${ }^{24} \mathrm{FH}$ also predicts a very high risk of cardiovascular disease (CVD) even in the absence of other traditional risk factors as patients possess these LDL-C concentrations from birth. ${ }^{25}$ Early diagnosis and treatment of FH with lipid-lowering therapy has proven to be both cost efficient and effective in mitigating cardiovascular morbidity and mortality risk. ${ }^{26}{ }^{27}$ Despite these benefits, numerous reports suggest that FH is currently underdiagnosed in the general population ${ }^{27}$ and that in some jurisdictions, a large proportion of affected individuals have difficulty accessing effective lipid-lowering therapies. ${ }^{28}$ Clinicians routinely consider estimates of disease prevalence, variations in different population groups (eg, age, sex, ethnicity) and the presence of known risk factors in formulating differential diagnoses. These factors also form important considerations when evaluating national strategies for the optimal identification and treatment of individuals. ${ }^{29}$ Thus, determining the prevalence of $\mathrm{FH}$ and its variation by sociodemographic factors provides an important first step in reducing disease burden.

While a number of narrative and systematic reviews have summarised studies of $\mathrm{FH}^{8}{ }^{813}{ }^{30-34}$ there has been no attempt to consolidate these studies to derive a robust prevalence estimate or to assess variation according to sociodemographic factors. We therefore aimed to systematically review the existing literature presenting estimates of $\mathrm{FH}$ in the adult general population and explore variation in prevalence estimates by age, sex, geographical location and study quality.

\section{METHODS}

We carried out a systematic review and meta-analysis in accordance with the Meta-analysis Of Observational Studies in Epidemiology consensus statement. ${ }^{35}$ The protocol for this review was registered with the PROSPERO International Prospective Register of Systematic Reviews (CRD42016042208).

\section{Study identification and selection}

This study was part of a series of systematic reviews with a standardised search strategy examining the disease burden posed by heterozygous $\mathrm{FH}$. We searched MEDLINE, EMBASE, PsycINFO, Global Health, the Cochrane Library and Pubmed (for publications ahead of print) for published, peer-reviewed literature using controlled vocabulary and keywords related to FH and relevant epidemiological terms. Results were limited to human studies published in English between 1 January 1990 and 31 January 2017. We reviewed reference lists of all included articles and relevant literature reviews, systematic reviews and meta-analyses for additional eligible studies. A detailed search strategy is included in the supplement to this manuscript (see online supplementary etable 1 ).

Titles and abstracts and full texts were evaluated in duplicate by independent reviewers (LEA, SDS) using standardised forms (see online supplementary etable 2). Disagreements were resolved through discussion to consensus. For inclusion in the systematic review of prevalence, studies were required to include live human participants and to report on the prevalence of FH. Studies were included if they ascertained FH frequency using one of the following methods (see online supplementary etables 3-5): (1) DNA-based evidence of LDLR, apolipoprotein-B (Apo B), or PCSK9 mutations; (2) Dutch Lipid Clinic Network (DLCN) criteria; (3) Simon Broome Registry (SBR) criteria; (4) Making Early Diagnosis to Prevent Early Death (MEDPED) criteria or (5) total cholesterol levels $(>290 \mathrm{mg} / \mathrm{dL}$ or $7.5 \mathrm{mmol} / \mathrm{L})$ or LDL-C levels $(>189 \mathrm{mg} / \mathrm{dL}$ or $4.9 \mathrm{mmol} / \mathrm{L}) .{ }^{34}$ We did not include articles reporting on the prevalence of or regional variations in specific LDLR, Apo B or PCSK9 mutations in study populations given their potential to underestimate FH frequencies.

\section{Data extraction}

One reviewer (LEA) independently extracted data regarding study characteristics (eg, design, population characteristics, diagnostic measures, prevalence estimates) from the full text of included articles. Another reviewer (RLR) checked the extracted data and any detected discrepancies were resolved. We did not attempt to contact authors of studies with missing or incomplete data nor did we exclude any such studies from our synthesis.

\section{Study quality assessment}

Two reviewers (LEA, RLR) independently assessed the quality of eligible studies using the Effective Public Health Practice Project (EPHPP) Quality Assessment Tool for Quantitative Studies (http://www.ephpp.ca/tools. html) and resolved discrepancies through consensus. It has been shown to be acceptable for use in evaluating a variety of study designs including randomised controlled trials, before-and-after studies and case control studies (see online supplementary etable 6). The tool assesses study quality across six domains: selection bias; study design; confounding variables; blinding protocols; data collection methods and handling of withdrawals and 
dropouts. Each dimension is rated on a three-point scale-strong, moderate and weak-and these ratings feed into a global rating of study quality. Global study quality is considered to be strong if none of the quality domains is rated as weak, moderate if one domain is rated as weak and weak if two or more domains are rated as weak.

\section{Data synthesis}

Our primary analysis consisted of a pooled estimate of prevalence across all studies using a random effects model. ${ }^{36}{ }^{37}$ We also pooled data from studies separately under the model in order to calculate the pooled prevalence of FH in children (ages $0-19)$ and adults ( $>20$ years of age). Where studies presented multiple diagnostic criteria, estimates derived from genetic testing were used in the analysis as this was thought to provide a more conservative estimate. Where studies derived estimates using DLCN criteria, we pooled reported cases of 'definite' or 'probable' FH to determine individual study estimates. Similarly, 'definite' or 'possible' FH diagnoses using Simon Broome criteria were pooled in the meta-analyses. Where multiple studies reported prevalence estimates from a single cohort, estimates were taken from the paper reporting the largest sample and the other paper excluded from the analysis. Potential influences on prevalence estimates were investigated using subgroup analyses and meta-regression. Where studies allowed, we descriptively compared prevalence estimates by age, sex, prevalence estimation method, study quality and geographical location within studies. We then assessed the influence of these factors on variation in the estimated prevalence using meta-regression models.

\section{Statistical analysis}

We calculated pooled prevalence figures with 95\% CIs using the DerSimonian and Laird random effects model. ${ }^{37}$ In meta-analyses of prevalence using inverse variance methods, when the frequency estimate of a single study approaches the limits of prevalence (ie, $0 \%$ or $100 \%$ of the population), the variance for that study moves toward 0 , leading to the resulting weight in the meta-analysis being overestimated. ${ }^{36}$ To accommodate for this, we conducted the meta-analysis with prevalence estimates that had been transformed using the double arcsine method. ${ }^{36}$ The final pooled result and 95\% CIs were then back transformed and expressed as percentages for ease of interpretation. We assessed heterogeneity in our pooled analyses using the $\mathrm{I}^{2}$ statistic as it is not sensitive to the scale of effect size or the total number of studies included in the meta-analysis. ${ }^{38}$ Finally, publication bias was examined formally using Egger's weighted regression, with significance set at $\mathrm{p}<0.10 .{ }^{39}$ Publication bias was also assessed visually using Begg's funnel plot as well as a Doi plot. ${ }^{40}{ }^{41}$ If publication bias was present, we used the trim and fill method to adjust for publication bias. ${ }^{40}$ Analyses were performed using the MetaXL add-in for Microsoft Excel (http://www.epigear.com). Forest plots were generated using DistillerSR Forest Plot Generator from Evidence Partners (https://www.evidencepartners.com/resources/forest-plot-generator/).

Meta-regression was used to discern the influence of age, sex, prevalence estimation method, study quality, geographical location, year of publication and study setting (ie, electronic health records versus general population registers) on our pooled prevalence estimate. We used Stata V.13.1 to perform the meta-regression analysis on the log scale of the back transformed effect size (ie, prevalence), with each trial weighting equal to that derived under the random effects model and between study variance estimated with the restricted maximum likelihood method. The log of the pooled prevalence estimate was used as the dependent variable whereas sample size, study quality scores, mean sample age and study proportions of female participants were used as continuous predictive variables. Categorical covariates such as prevalence estimation method and geographical location were dummy-coded and examined through a joint test for all dummy-coded covariates.

\section{Sensitivity analyses}

We conducted additional analyses to assess the robustness of our pooled prevalence estimate. We examined the impact of time on the diagnosis of FH by sequentially excluding studies published before the year 2000 and 2010. We also assessed the impact of study setting by comparing estimates derived from population-based databases with those in patient cohorts (ie, community clinics, patient registries, electronic health records). Finally, we excluded studies using LDL-C to diagnose FH as well as those from countries with known founder populations as both were likely to result in a higher pooled frequency.

\section{RESULTS}

\section{Study selection}

Our search identified 4153 citations, of which 3574 were unique. After applying our inclusion and exclusion criteria, 90 articles progressed to screening at the full-text level, of which 21 articles were included in this review. The flow of included studies is presented in figure 1.

\section{Characteristics of included studies}

Twenty-one studies estimating point prevalence of $\mathrm{FH}$ were included in this review (table 1). The majority of these studies were European $(n=9)$, while others were conducted in North America $(n=4)$, Asia $(n=2)$, Australia $(n=3)$ and Africa $(n=1)$. Two of the studies pooled data from international cohorts. ${ }^{10}{ }^{42}$ Combined, they represented data from 28 countries across four continents. Studies representing multiple countries included data from coronary artery disease $^{10}$ and dyslipidaemia cohorts. ${ }^{42} \mathrm{FH}$ is overexpressed among those with coronary heart disease as well as statin-treated individuals. ${ }^{24}$ For these reasons, we elected against pulling country-specific data from these papers. Among all included studies, females comprised between 


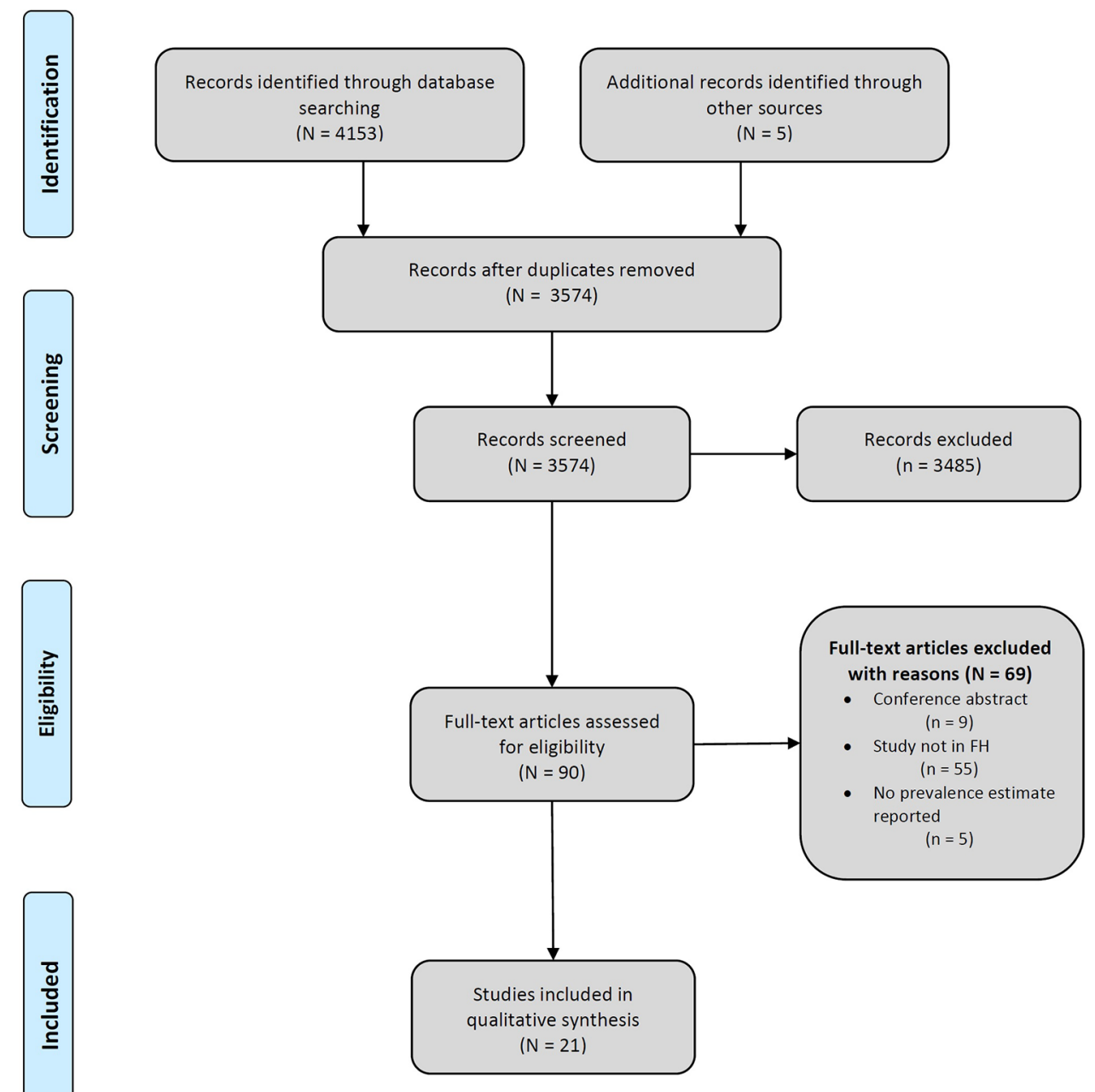

Figure 1 Flow of studies included in systematic review of heterozygous familial hypercholesterolaemia prevalence. FH, familial hypercholesterolaemia.

$26.4 \%$ and $55.0 \%$ of the total sample. Four studies diagnosed FH using DLCN criteria, ${ }^{42-45}$ three studies used genetic sequencing, ${ }^{46-48}$ three studies used LDL-C measurements, ${ }^{4-51}$ one study used SBR criteria ${ }^{7}$ and one employed MEDPED criteria. ${ }^{6}$ Another four included studies reported prevalence estimates using more than one method for comparison. ${ }^{10115253}$ Prevalence estimates reported in individual studies ranged from $0.05 \%$ (95\% CI $0.05 \%$ to $0.06 \%)$ to $5.62 \%$ (95\% CI $5.44 \%$ to $5.79 \%$ ). When evaluated by the EPHPP tool, most studies were rated as being moderate $(\mathrm{n}=7)$ or strong $(\mathrm{n}=13)$ in quality. On EPHPP domains, studies were most likely to receive weak ratings due to a low likelihood of representing the general population, a failure to account for missing participant data or adjust for relevant confounders (see online supplementary etable 7).

\section{Meta-analysis}

\section{Overall pooled prevalence}

Nineteen estimates were included in the meta-analysis of overall prevalence, representing 2458456 unique individuals. ${ }^{6} 71042-48505153-58$ A further two studies reported data from cohorts represented by other studies within a shorter sampling frame, creating the potential for the overlap of cohorts. ${ }^{11} 49$ These estimates were excluded to avoid overweighting a population. The overall random effects pooled prevalence of $\mathrm{FH}$ was $0.40 \%(95 \%$ CI $0.29 \%$ to $0.52 \%$ ) (figure 2 ).

\section{Prevalence of $\mathrm{FH}$ in adults}

Sixteen prevalence estimates were included in the meta-analysis of adult prevalence, representing 2431053 unique individuals. ${ }^{6}{ }^{7} 10$ 42-48 53-57 The overall random effects pooled prevalence of $\mathrm{FH}$ was $0.40 \% \quad(95 \%$ CI $0.29 \%$ to $0.54 \%$ ) (see online supplementary etable 8 ).

\section{Prevalence of $\mathrm{FH}$ in children}

Combining four studies ( $\mathrm{n}=27403)$ which reported FH prevalence estimates in individuals aged under 19 (see online supplementary etable 9), we calculated a pooled prevalence of $0.36 \%$ ( $95 \%$ CI $0.28 \%$ to $0.45 \%$ ), with little heterogeneity $\left(\mathrm{I}^{2}=13.32 \%\right) .{ }^{43} 505158$

Variation in prevalence by age

Six studies 71143495355 reported age-stratified data on the adult prevalence of $\mathrm{FH}$, but only two of these presented data in forms amenable for pooled analysis (figure 3). ${ }^{753}$ All studies showed variation in FH frequency with age, 


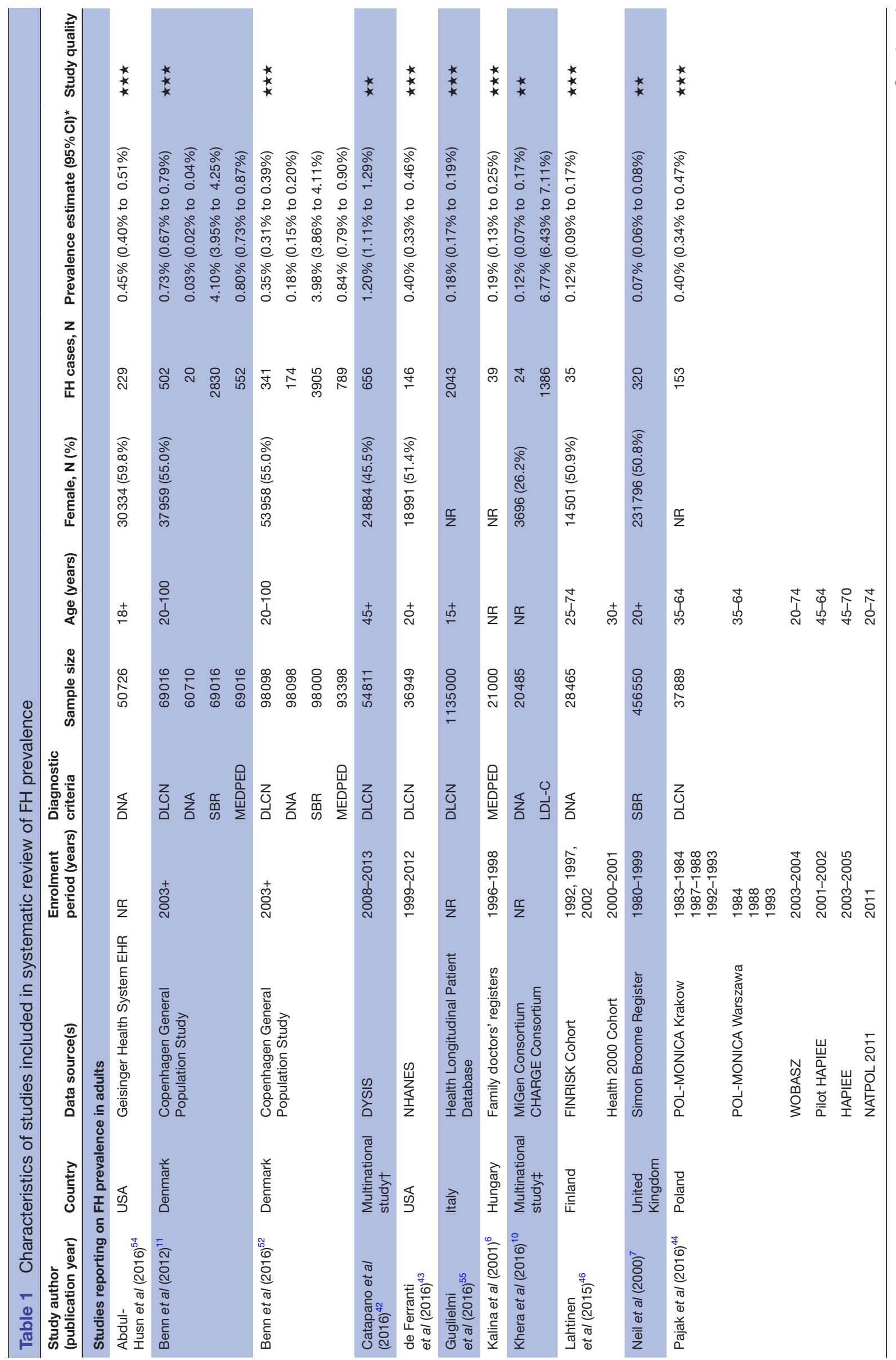



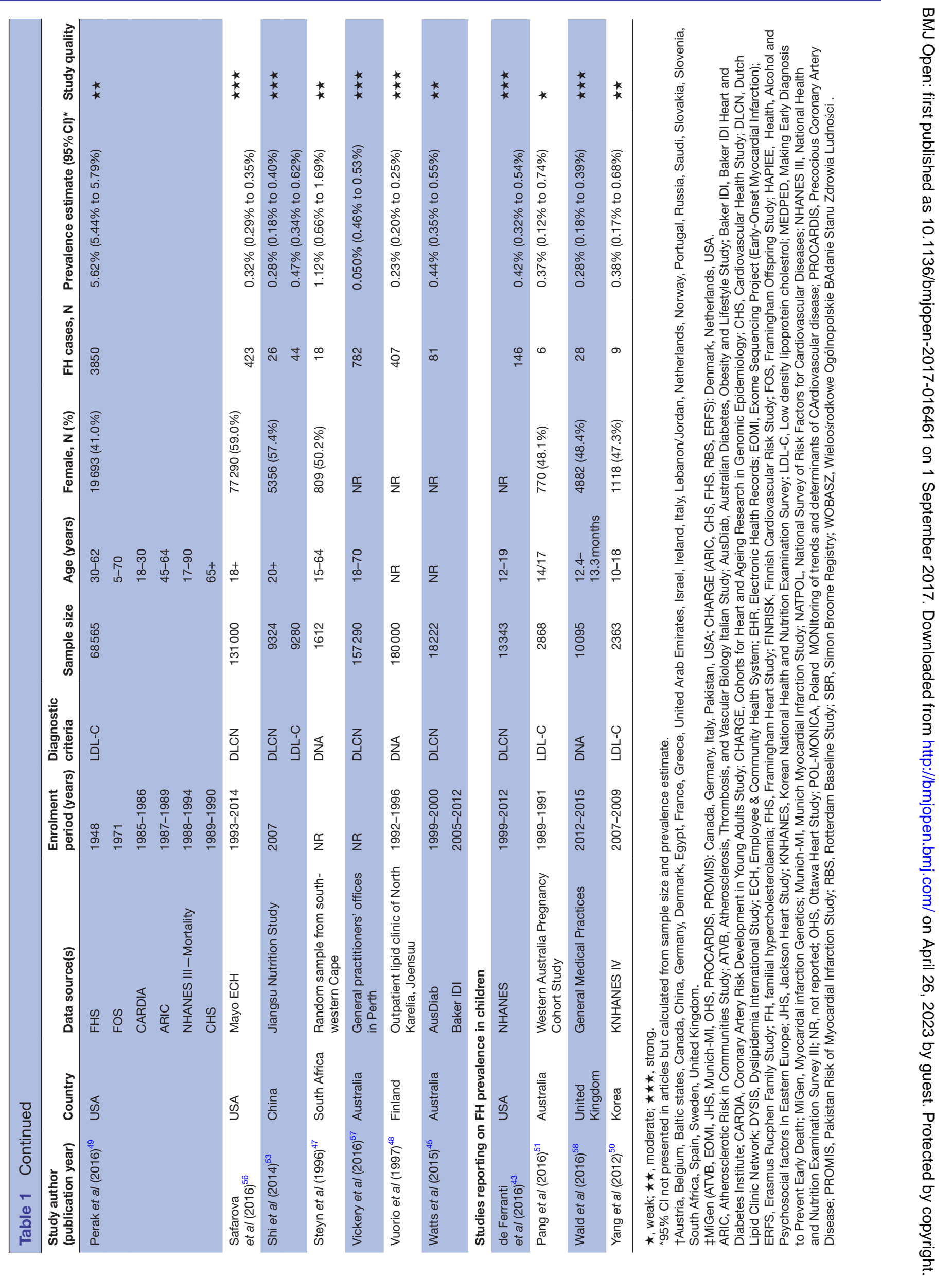


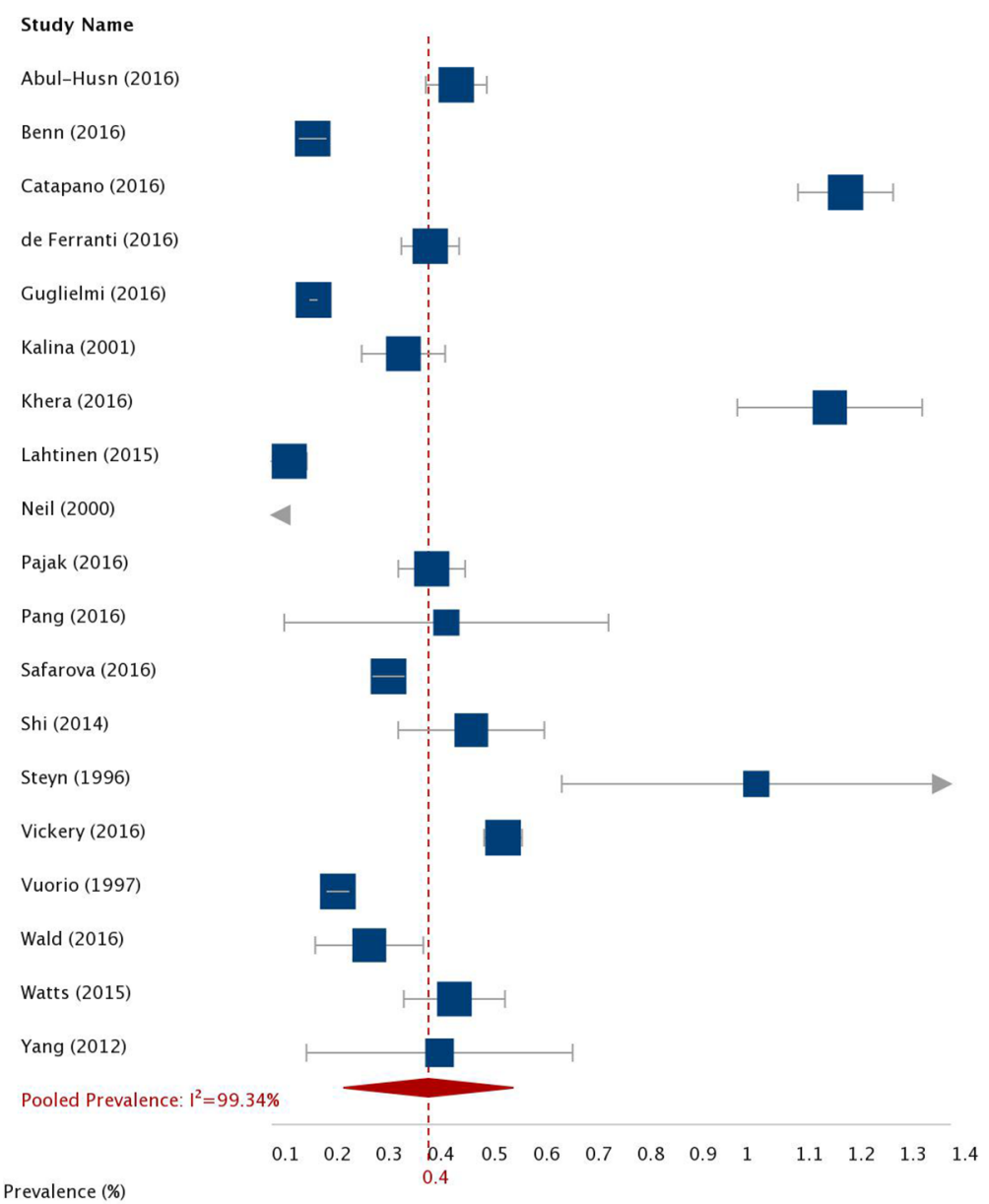

\begin{tabular}{|c|c|c|c|c|}
\hline POP & PREV & LCL & UCL & WGHT \\
\hline 50726 & 0.45 & 0.39 & 0.51 & $5.53 \%$ \\
\hline 98098 & 0.18 & 0.15 & 0.2 & $5.56 \%$ \\
\hline 54811 & 1.2 & 1.11 & 1.29 & $5.53 \%$ \\
\hline 50292 & 0.4 & 0.35 & 0.46 & $5.53 \%$ \\
\hline 1135000 & 0.18 & 0.17 & 0.19 & $5.6 \%$ \\
\hline 21000 & 0.35 & 0.27 & 0.43 & $5.43 \%$ \\
\hline 14117 & 1.16 & 0.99 & 1.35 & $5.35 \%$ \\
\hline 28465 & 0.12 & 0.09 & 0.17 & $5.47 \%$ \\
\hline 456550 & 0.07 & 0.06 & 0.08 & $5.59 \%$ \\
\hline 37889 & 0.4 & 0.34 & 0.47 & $5.5 \%$ \\
\hline 1602 & 0.37 & 0.12 & 0.74 & $3.95 \%$ \\
\hline 131000 & 0.32 & 0.29 & 0.35 & $5.57 \%$ \\
\hline 9324 & 0.47 & 0.34 & 0.62 & $5.23 \%$ \\
\hline 1612 & 1.12 & 0.66 & 1.69 & $3.96 \%$ \\
\hline 157290 & 0.54 & 0.51 & 0.58 & $5.58 \%$ \\
\hline 180000 & 0.23 & 0.2 & 0.25 & $5.58 \%$ \\
\hline 10095 & 0.28 & 0.18 & 0.39 & $5.25 \%$ \\
\hline 18222 & 0.44 & 0.35 & 0.55 & $5.4 \%$ \\
\hline 2363 & 0.38 & 0.17 & 0.68 & $4.37 \%$ \\
\hline 2458456 & 0.4 & 0.29 & 0.52 & $100 \%$ \\
\hline
\end{tabular}

Figure 2 Forest plot of overall pooled prevalence (\%) of heterozygous familial hypercholesterolaemia. $I^{2}$, between-study heterogeneity; LCL, lower confidence limit; POP, population; PREV, prevalence; UCL, upper confidence limit; WGHT, weight under the random-effects model. Note: prevalence estimates were derived using the double-arcsine method, back-transformed and expressed as percentages for ease of interpretation.

with an increase in prevalence that peaked between ages 60 and 69 and declining thereafter, a trend reflected in our pooled estimates.

\section{Variation in prevalence by sex}

Nine studies presented prevalence figures by sex, ${ }^{7} 1042-4446475253$ most of which reported similar FH frequencies between men and women. Our pooled prevalence estimates (figure 4) were comparable between males $(0.42 \% ; 95 \%$ CI $0.18 \%$ to $0.75 \% ; \mathrm{n}=364130)$ and females $(0.45 \%$; $95 \%$ CI $0.19 \%$ to $0.82 \%$; $\mathrm{n}=319726)$ (OR: $0.85 ; 95 \%$ CI 0.0 .69 to $1.07 ; \mathrm{n}=639717$ ).

\section{Variation in prevalence by geographic location}

When FH was analysed by continent (figure 5), European (seven studies; $\mathrm{n}=1957002$ ) and Asian studies (one study; $\mathrm{n}=9324$ ) tended to report lower prevalence estimates than our overall pooled prevalence estimate, while North American (three studies; $\mathrm{n}=236537$ ) and Australasian (two studies; $\mathrm{n}=175512$ ) studies reported estimates comparable to it. The one study from South Africa $(n=1612)$ reported a greater pooled FH prevalence than our pooled estimate, as did studies of international cohorts.
Variation in prevalence by diagnostic criteria

Frequencies from studies in the DNA-based analysis subgroup were comparable to the pooled prevalence estimate $(0.40 \% ; 95 \%$ CI $0.24 \%$ to $0.58 \%)$ while DLCN $(0.46 \%$; $95 \%$ CI $0.25 \%$ to $0.70 \%)$ and LDL-C-based estimates $(0.45 \% ; 95 \% \mathrm{CI} 0.34 \%$ to $0.57 \%)$ tended to report slightly higher frequencies (see online supplementary efigure 1). Of two studies exclusively using $\mathrm{SBR}^{7}$ or MEDPED $^{6}$ criteria, both reported lower frequencies than our pooled prevalence estimate.

Variation in prevalence by study quality

When stratified by study quality ratings, studies rated strong had a lower estimate of $\mathrm{FH}$ prevalence with greater precision $(0.33 \%$; $95 \%$ CI $0.24 \%$ to $0.43 \%)$ than studies rated moderate in quality $(0.75 \% ; 95 \%$ CI $0.29 \%$ to $1.29 \%)$ or low quality $(0.37 \%, 95 \%$ CI $0.12 \%$ to $0.74 \%)$ (see online supplementary efigure 2 ).

\section{Meta-regression analyses}

Considerable heterogeneity existed between studies ( ${ }^{2}: 99.34 \%$; $95 \%$ CI $99.24 \%$ to $\left.99.44 \%\right)$. The results of eight meta-regression analyses (table 2) showed little evidence of an effect of age $(\mathrm{p}=0.79)$, sex $(\mathrm{p}=0.17)$, 


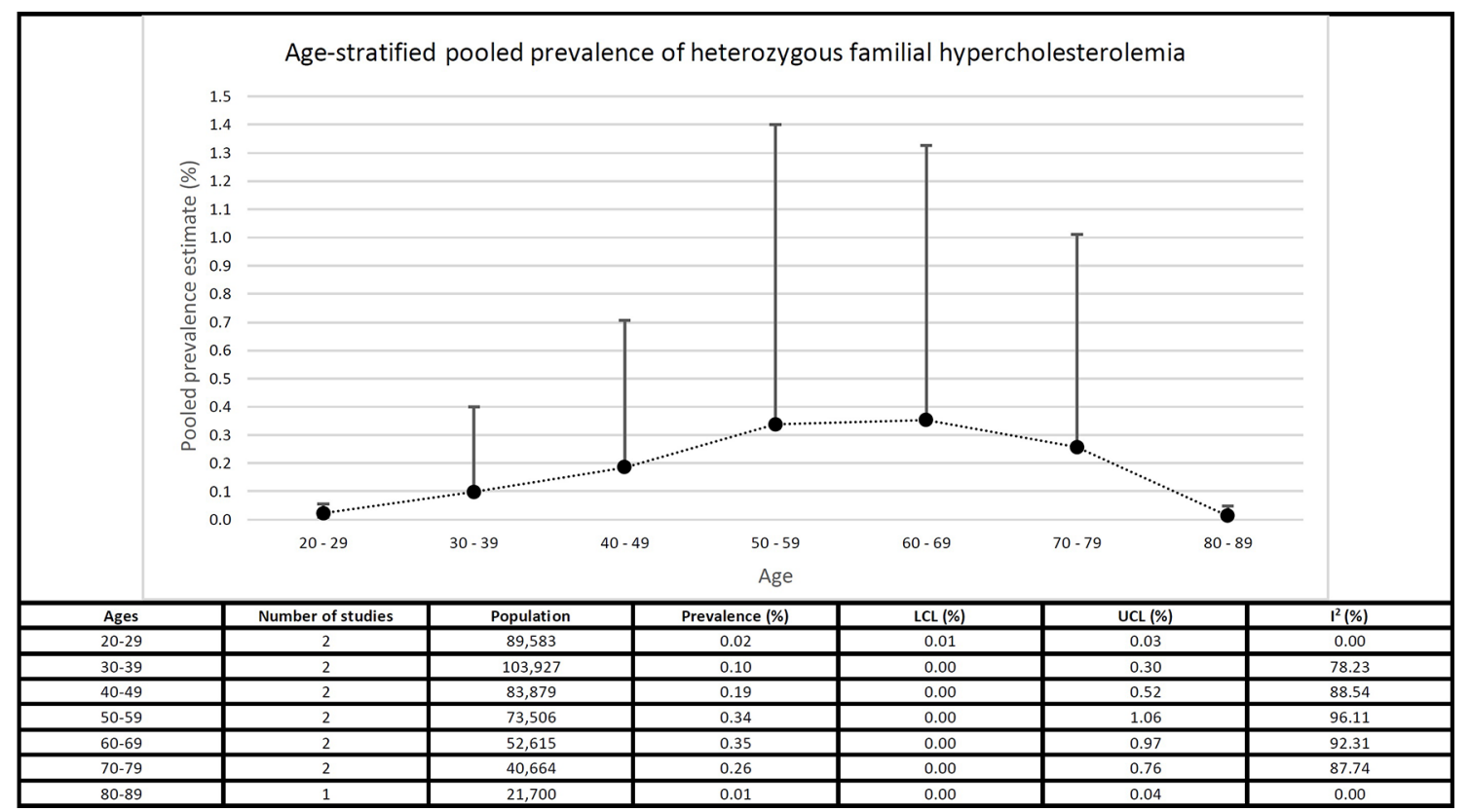

Figure 3 Age-stratified pooled familial hypercholesterolaemia $(\mathrm{FH})$ prevalence estimates and $95 \% \mathrm{Cls}$. figure 3 Error bars are representative of $95 \%$ Cls for each pooled estimate. Lower Cls are omitted; all cross $0 \%$. $I^{2}$, between-study heterogeneity; LCL, lower confidence limit; UCL, upper confidence limit.

sample size $(p=0.06)$, diagnostic criteria $(\mathrm{p}=0.23)$, study setting $(\mathrm{p}=0.50)$, quality $(\mathrm{p}=0.82)$ or year of publication $(\mathrm{p}=0.52)$ on our pooled prevalence estimate. Joint meta-regression tests showed significant differences in prevalence estimates among categories of studies when stratified by geographical location $(\mathrm{p}=0.04)$. Major asymmetry was present in both Begg's funnel plot and the Doi plot (see online supplementary efigure 3 ) and the results of Egger's test suggested that publication bias may have been present $(p<0.001) .{ }^{59}$ When we used the trim and fill method to control for publication bias, nine additional studies were generated with estimates comparable to or lower than our pooled prevalence estimate, bringing the pooled prevalence of $\mathrm{FH}$ to $0.20 \%$ (95\% CI $0.10 \%$ to $0.40 \%)$.

\section{Sensitivity analyses}

Pooled prevalence estimates were broadly consistent in seven sensitivity analyses (see online supplementary etable 10). Studies estimating $\mathrm{FH}$ prevalence in patient cohorts ( $0.33 \%$; $95 \%$ CI $0.21 \%$ to $0.47 \%)$ tended to report lower frequencies than those in large population-based samples $(0.45 \% ; 95 \%$ CI $0.26 \%$ to $0.68 \%)$. Heterogeneity of these estimates was significant and comparable $(>99 \%)$.

\section{DISCUSSION}

Our meta-analysis of 19 cohort studies including 2458456 unique individuals found an $\mathrm{FH}$ prevalence of $0.40 \%$ in the general population. This suggests that as many as 1 in 250 individuals may be affected by $\mathrm{FH}$ (95\% CI 1 in 345 to 1 in 192), equating to nearly 30 million people worldwide. ${ }^{60}$ This is a higher frequency than observed in prior reports and supports current thinking that FH is underdiagnosed, and thus likely undertreated in the general population. ${ }^{61}$ This is further supported by sensitivity analyses in which patient cohort studies were found to report lower prevalence estimates than those using large population databases.

Interestingly, we detected a slightly lower prevalence of FH in those aged 0-19 (1 in 278; 95\% CI 1 in 345 to 1 in 222). Further, FH prevalence tended to increase with age. This trend runs counterintuitively to expectations given that FH is a genetic condition with a high risk of CVD-related mortality-frequency estimates should be comparable in adults and children save for age-related declines in prevalence associated with premature mortality. Our findings may be explained by insufficient dyslipidaemia screening in children and adolescents. ${ }^{62-64}$ Indeed, follow-up data from the Simon Broome FH registry, following more than 300000 patients found that only a quarter of affected patients received diagnoses by middle age, with the highest rates of underdiagnosis among children and adolescents. ${ }^{7}$ However, LDL-C levels also rise with age, making it likely for older individuals to be diagnosed using established clinical criteria. It remains possible that the disparity in prevalence may be due to the inability of population-based studies to account for age-related increases in LDL-C and the reduced sensitivity this confers in detecting $\mathrm{FH}^{65}$

Our finding that $\mathrm{FH}$ affects males and females equally has important implications. Many cases of FH are diagnosed following the first cardiac event, which has a later onset for women relative to men. ${ }^{27}$ This makes it possible that women with FH may go unrecognised for longer. Yet, more women may be expected to qualify for diagnosis using clinical characteristics at later ages, primarily due 

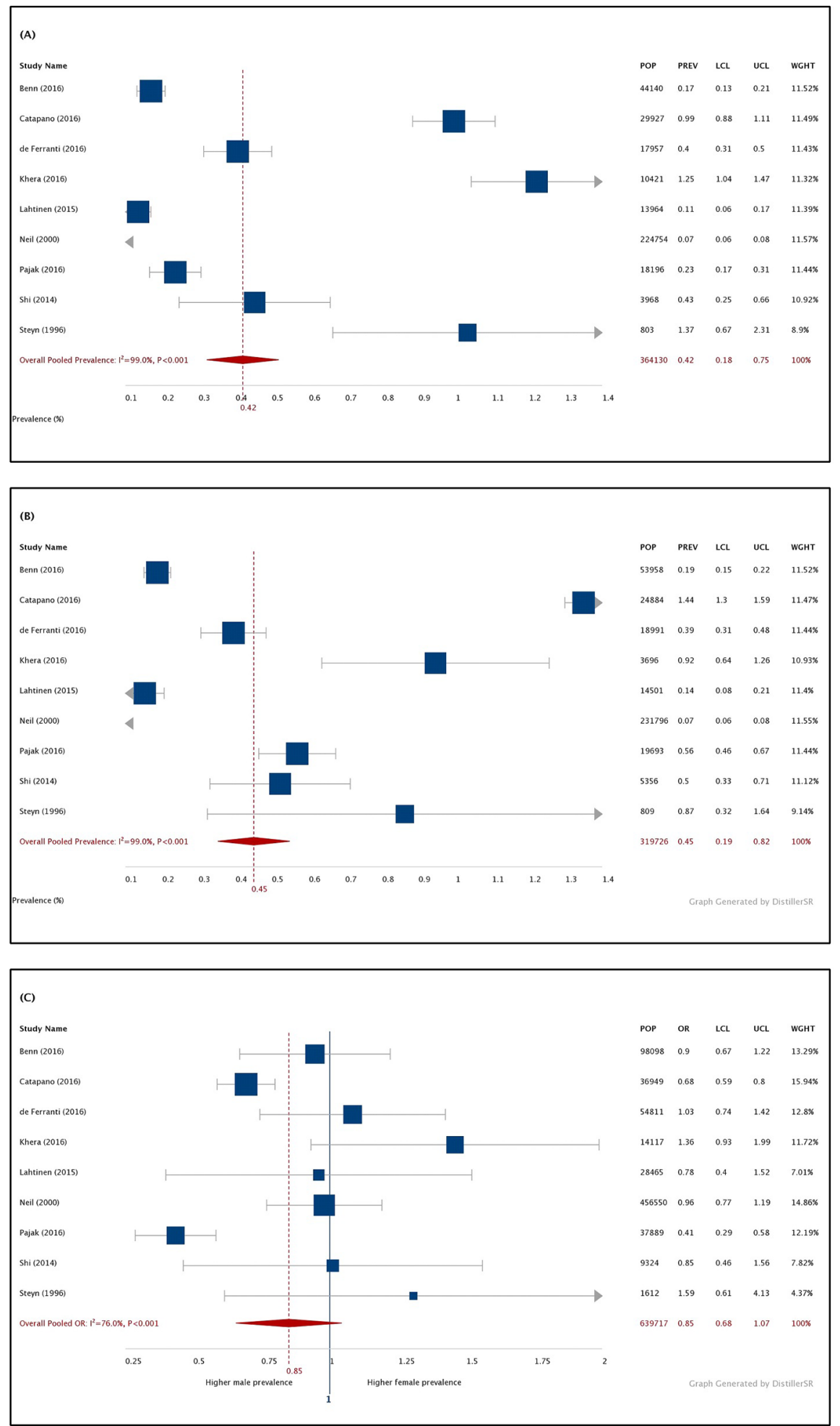

Figure 4 (A) Forest plot of pooled prevalence (\%) of heterozygous FH in the male population. (B) Forest plot of pooled prevalence (\%) of $\mathrm{FH}$ in the female adult population. (C) Forest plot of pooled OR of male:female $\mathrm{FH}$ prevalence. $\mathrm{FH}$, familial hypercholesterolaemia; $I^{2}$, between-study heterogeneity; LCL, lower confidence limit; POP, population; PREV, prevalence; UCL, upper confidence limit; WGHT, weight under the random-effects model. Note: prevalence estimates were derived using the double-arcsine method, back-transformed and expressed as percentages for ease of interpretation.

to the delayed onset of coronary artery disease. Whether delayed $\mathrm{FH}$ detection in women relative to men confers poorer clinical outcomes has yet to be formally explored in the literature. However, one of our included studies observed that after age 60, higher proportions of women met criteria for an FH diagnosis, suggesting that many men with $\mathrm{FH}$ had died at an earlier age. ${ }^{11}$ Identifying sex-related differences in $\mathrm{FH}$ presentation may allow for 


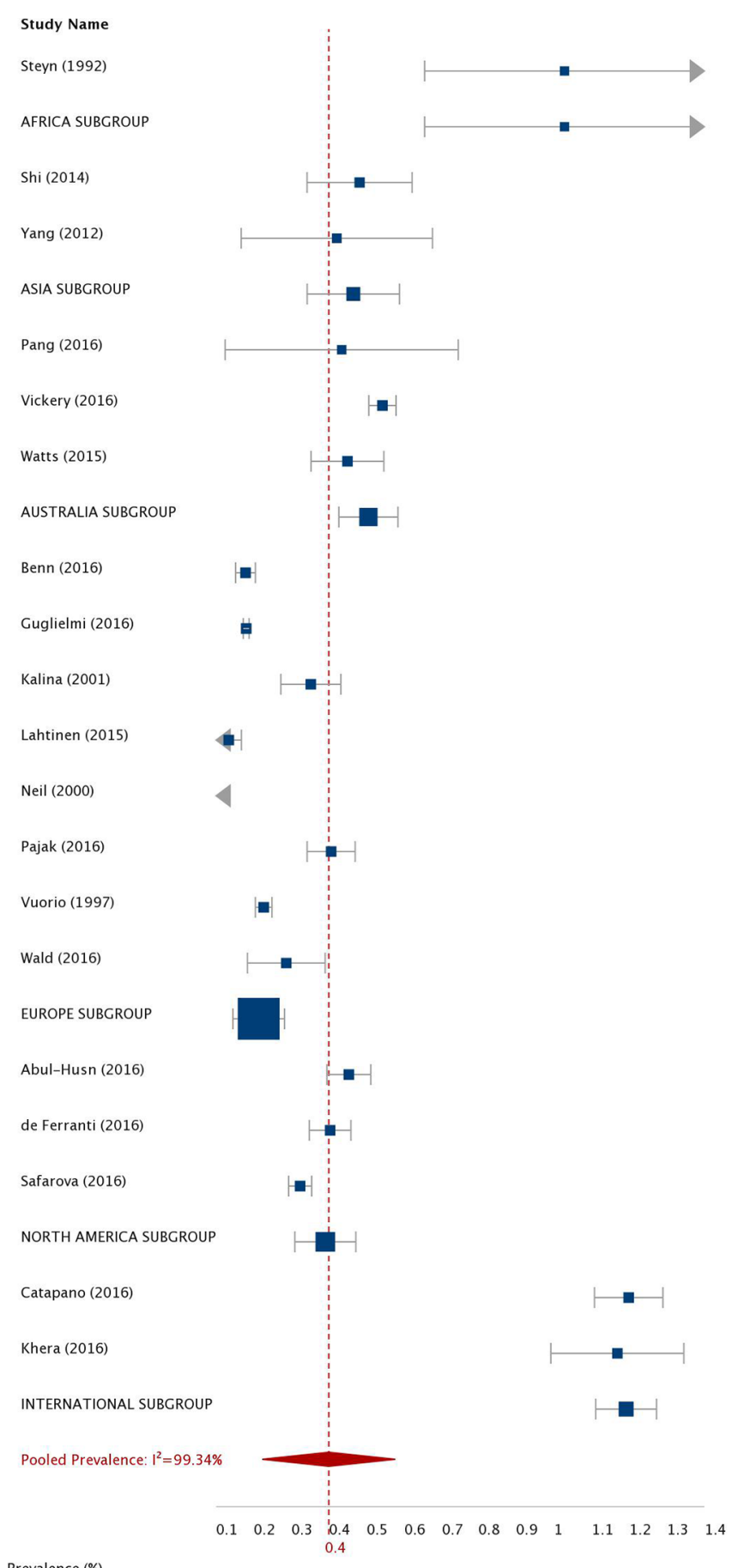

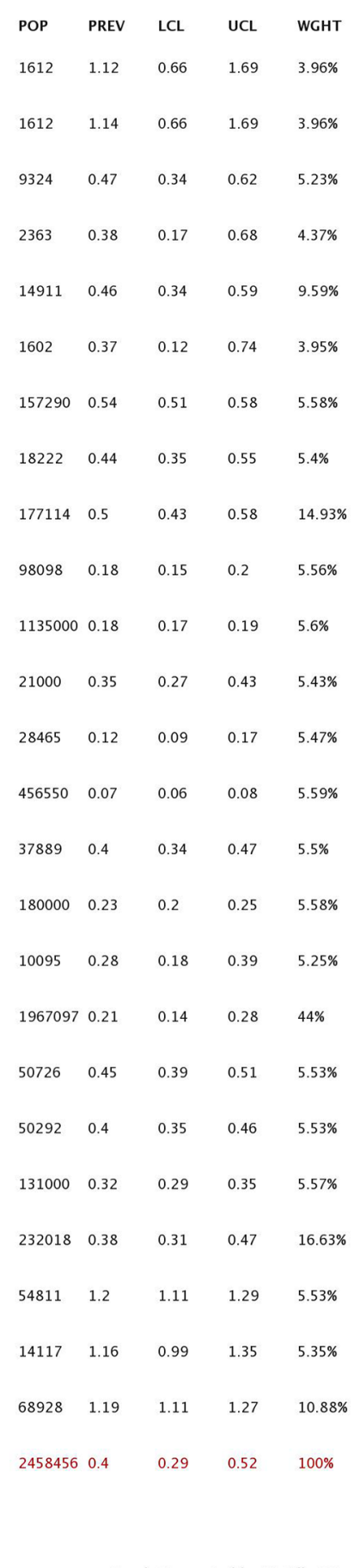

Graph Generated by DistillerSR

Figure 5 Forest plot of overall pooled prevalence (\%) of heterozygous familial hypercholesterolaemia stratified by population geography. $\mathrm{I}^{2}$, between-study heterogeneity; LCL, lower confidence limit; POP, population; PREV, prevalence; UCL, upper confidence limit; WGHT, weight under the random-effects model. Note: prevalence estimates were derived using the doublearcsine method, back-transformed and expressed as percentages for ease of interpretation.

earlier FH diagnosis and represents an important clinical priority. New diagnostic criteria developed through improved use of routinely collected health data may make this possible. ${ }^{66}$

We also found lower prevalence reports in Europe relative to regions elsewhere. Thus far, much of the regional variation in $\mathrm{FH}$ prevalence has been attributed to the presence of founder populations. Founder effects occur when subpopulations are formed by the immigration of 'founder subjects', leading to a higher proportion of individuals who share a mutation in subsequent generations due to genetic drift. ${ }^{13}$ Though influenced by a predominance of European studies, our review suggests the potential for variations in FH frequency between countries extending beyond founder effects. This is important given that for many of the world's countries, rates of FH still remain unknown. This includes North America, where studies from USA comprise the evidence 
Table 2 Meta-regression analyses for pooled estimate of familial hypercholesterolaemia prevalence

\begin{tabular}{llllllc}
\hline Covariate & Observations & Coefficient & $\mathbf{9 5 \%} \mathbf{C l}$ & $\mathbf{p}$ Value & Adjusted $\mathbf{R}^{\mathbf{2}}$ (\%) & $\mathbf{I}^{\mathbf{2}}$ residual (\%) \\
\hline Age & 11 & $8.26 \times 10^{-3}$ & -0.06 to 0.08 & 0.79 & -10.29 & 99.65 \\
Diagnostic criteria & 15 & NA & NA & 0.23 & 12.77 & 99.45 \\
\hline Geographical location & 19 & NA & NA & 0.04 & 75.92 & 99.00 \\
Sex & 13 & -4.07 & -10.18 to 2.00 & 0.17 & 8.99 & 99.67 \\
Sample size & 19 & $-1.21 \times 10^{-6}$ & $-2.47 \times 10^{-6}$ to & 0.06 & 4.20 & 100.00 \\
Study quality & 19 & 0.02 & -0.16 to 0.20 & 0.82 & -5.64 & 99.54 \\
Study setting & 19 & 0.24 & -0.49 to 0.96 & 0.50 & -2.65 & 99.28 \\
Year of publication & 19 & 0.16 & -0.04 to 0.07 & 0.52 & -2.54 & 99.41 \\
\hline
\end{tabular}

${ }^{*} \mathrm{p}<0.05$.

Adjusted $\mathrm{R}^{2}$, proportion of between-study variance explained with Knapp-Hartung modification; $I^{2}$ residual, per cent residual variation due to heterogeneity; NA, not applicable; Observations, number of studies with observations included in the meta-regression model.

base for ascertaining study prevalence. CVD remains the leading cause of death worldwide ${ }^{67}$ and, left untreated, nearly $85 \%$ of males and $50 \%$ of females with $\mathrm{FH}$ are expected to suffer coronary events prior to age $65 .{ }^{27}$ Thus, greater efforts should be made to explore region-specific frequencies of $\mathrm{FH}$ prevalence and more accurately characterise disease burden. Accurate prevalence estimates, augmented by recent big data approaches and the introduction of International Classification of Diseases, 10th Revision codes for FH should facilitate increased awareness and improved management.

How FH should be identified remains an area of continued debate. A number of organisations have recommended universal lipid screening in childhood as a strategy to identify $\mathrm{FH}^{68-70}$ However, a recent report by the US Preventive Services Task Force concluded that there was 'inadequate direct evidence on the benefit of screening for $\mathrm{FH}{ }^{71}$ In addition, these programmes come with the added risks of potential overdiagnosis, fiscal and non-fiscal health system burden and adverse psychosocial impacts for children and families. ${ }^{71}$ As an alternative, some European countries have developed genetic FH screening strategies. However, such programmes are neither currently universally accessible nor deemed to be cost-effective. ${ }^{81-23}$ DNA-based identification may also fail to capture individuals with undiscovered mutations or those with polygenic forms of FH that still demonstrate the clinical phenotype. ${ }^{72}$ Finally, the diagnostic accuracy of these programmes has been challenged by findings that up to $30 \%$ of estimated cases may not be identified in countries with some of the most robust screening programmes, due to lack of index cases to inform cascade screening. ${ }^{73}$ In light of these limitations, the high degree of concordance between our pooled prevalence estimates derived through DLCN and DNA-based analyses are clinically important. Due to a simplified approach-facilitated by the use of readily observable clinic characteristics and biochemical parameters-DLCN criteria may facilitate the more ready identification of patients affected by $\mathrm{FH}$ in primary care. Though other clinical criteria may have comparable clinical utility, our study currently provides insufficient evidence in strong support of them. Regardless, improving the identification of $\mathrm{FH}$ and mitigating CVD and mortality requires a multifaceted approach involving clinical, biochemical and genetic parameters.

These findings provide new insights into FH prevalence. Yet, they should be interpreted in light of some important limitations. First, despite an extensive search strategy, we included only peer-reviewed English language studies indexed in six online databases and it remains possible that other relevant studies went unpublished or were indexed in other languages, in print repositories or within the grey literature. ${ }^{74}$ Second, we did not contact study authors for additional data or clarifications of their published studies. While this was counterbalanced in part by the use of a tool with high inter-rater agreement for quality assessment, ${ }^{75}$ agreement levels between reviewers and authors have yet to be explored with the EPHPP tool. Third, while geographical location of our included studies was significantly associated with variance in FH prevalence, our analyses possessed a considerable amount of between-study heterogeneity, the majority of which remains unexplained. This may be attributed to limited power in our meta-regression analyses due to small numbers of observations. ${ }^{38}$ In which case, our subgroup analyses provide more credible insight into the sociodemographic variation of $\mathrm{FH}$ prevalence though even these are limited by the lack of interaction tests in our subgroup analyses. It is important to note that the high degree of heterogeneity in our meta-analyses does not imply imprecision in our prevalence estimate. ${ }^{38}$ Indeed, a key strength of our study is its sample size and the greater power and precision it conferred to our analyses. The heterogeneity between studies are thus more likely reflective of real differences in study populations, designs and outcome measurements. ${ }^{36}$ This heterogeneity was anticipated and accommodated for through random effects meta-analysis. 


\section{CONCLUSION}

Our systematic review found that FH currently affects 1 in 250 people in the adult population. While $\mathrm{FH}$ affects males and females equally, regional and age-specific variations exist in $\mathrm{FH}$ frequency. With the range of treatment options available for this condition increased, particularly with the recent advent of PCKS9 inhibitors, greater efforts should be made to identify individuals who could stand to benefit from therapy.

Acknowledgements The authors would like to thank staff at the Institute for Clinical Evaluative Sciences for logistical support of this systematic review.

Contributors LEA conceived and designed the study, conducted the study, provided methodological support, conducted the analyses, interpreted the results and wrote, read and edited the manuscript. JG interpreted the results, read and edited the manuscript. SDS, RLR and JMA conducted the study and read and edited the manuscript. AC conceived and designed the study, provided methodological support and read and edited the manuscript. JVT conceived and designed the study, provided methodological support, interpreted the results, guided the analysis and read and edited the manuscript.

Funding LEA was supported by the Comprehensive Research Education for Medical Students Scholar Program at the University of Toronto. JG holds the McGill/ Novartis Chair at McGill University. JVT is funded by a Canada Research Chair in Health Services Research from the Canadian Institutes of Health Research (CIHR) and an Eaton Scholar Award from the Department of Medicine at the University of Toronto. This research is also funded by an Institute of Circulatory and Respiratory Health-Canadian Institutes of Health Research Chronic Diseases Team grant (no. TCA 118349) to the Cardiovascular Health in Ambulatory Care Research Team (http://www.canheart.ca) and the Institute for Clinical Evaluative Sciences (ICES), which is funded by an annual grant from the Ontario Ministry of Health and LongTerm Care (MOHLTC). The funders had no role in study design, data collection and analysis, decision to publish or preparation of the manuscript.

Competing interests None declared.

Provenance and peer review Not commissioned; externally peer reviewed. Data sharing statement Additional data are presented in supplemental files. Open Access This is an Open Access article distributed in accordance with the Creative Commons Attribution Non Commercial (CC BY-NC 4.0) license, which permits others to distribute, remix, adapt, build upon this work non-commercially, and license their derivative works on different terms, provided the original work is properly cited and the use is non-commercial. See: http://creativecommons.org/ licenses/by-nc/4.0/

(C) Article author(s) (or their employer(s) unless otherwise stated in the text of the article) 2017. All rights reserved. No commercial use is permitted unless otherwise expressly granted.

\section{REFERENCES}

1. Goldstein JL, Schrott HG, Hazzard WR, et al. Hyperlipidemia in coronary heart disease. II. genetic analysis of lipid levels in 176 families and delineation of a new inherited disorder, combined hyperlipidemia. J Clin Invest 1973;52:1544-68.

2. Slack J. Inheritance of familial hypercholesterolemia. Atheroscler Rev 1979;5:35-66.

3. Heiberg A, Berg K, are BK. The inheritance of hyperlipoproteinaemia with xanthomatosis:a study of 132 kindreds. Clin Genet 1976;9:203-33.

4. Andersen GE, Lous P, Friis-Hansen B. Screening for hyperlipoproteinemia in 10,000 danish newborns. Follow-up studies in 522 children with elevated cord serum VLDL-LDL-cholesterol. Acta Paediatr Scand 1979;68:541-5.

5. Mabuchi H, Haba T, Ueda K, et al. Serum lipids and coronary heart disease in heterozygous familial hypercholesterolemia in the Hokuriku district of Japan. Atherosclerosis 1977;28:417-23.

6. Kalina A, Császár A, Czeizel AE, et al. Frequency of the R3500Q mutation of the apolipoprotein B-100 gene in a sample screened clinically for familial hypercholesterolemia in Hungary. Atherosclerosis 2001;154:247-51.
7. Neil HA, Hammond T, Huxley R, et al. Extent of underdiagnosis of familial hypercholesterolaemia in routine practice: prospective registry study. BMJ 2000;321:148.

8. Marks D, Wonderling D, Thorogood M, et al. Cost effectiveness analysis of different approaches of screening for familial hypercholesterolaemia. BMJ 2002;324:1303.

9. Trikalinos TA, Salanti G, Khoury MJ, et al. Impact of violations and deviations in Hardy-Weinberg equilibrium on postulated genedisease associations. Am J Epidemiol 2006;163:300-9.

10. Khera AV, Won HH, Peloso GM, et al. Diagnostic yield and clinical utility of sequencing familial hypercholesterolemia genes in patients with severe hypercholesterolemia. J Am Coll Cardiol 2016;67:2578-89.

11. Benn M, Watts GF, Tybjaerg-Hansen A, et al. Familial hypercholesterolemia in the Danish general population: prevalence, coronary artery disease, and cholesterol-lowering medication. J Clin Endocrinol Metab 2012;97:3956-64.

12. Goldberg AC, Gidding SS. Knowing the prevalence of familial hypercholesterolemia matters. Circulation 2016;133:1054-7.

13. Austin MA, Hutter CM, Zimmern RL, et al. Genetic causes of monogenic heterozygous familial hypercholesterolemia: a HuGE prevalence review. Am J Epidemiol 2004;160:407-20.

14. Patterson D, Slack J. Lipid abnormalities in male and female survivors of myocardial infarction and their first-degree relatives. Lancet 1972;1:393-9.

15. Moorjani S, Roy M, Gagné C, et al. Homozygous familial hypercholesterolemia among french Canadians in Québec province. Arteriosclerosis 1989;9:211-6.

16. Slimane MN, Pousse H, Maatoug F, et al. Phenotypic expression of familial hypercholesterolaemia in central and Southern Tunisia. Atherosclerosis 1993;104:153-8.

17. Seftel HC, Baker SG, Sandler MP, et al. A host of hypercholesterolaemic homozygotes in South Africa. Br Med J 1980;281:633-6.

18. Seftel HC, Baker SG, Jenkins T, et al. Prevalence of familial hypercholesterolemia in Johannesburg Jews. Am J Med Genet 1989;34:545-7

19. Rubinsztein DC, van der Westhuyzen DR, Coetzee GA, et al. Monogenic primary hypercholesterolaemia in South Africa. S Afr Med J 1994;84:339-44.

20. Hegele RA. Improving the monitoring and care of patients with familial hypercholesterolemia. J Am Coll Cardiol 2016;67:1286-8.

21. Oliva J, López-Bastida J, Moreno SG, et al. [Cost-effectiveness analysis of a genetic screening program in the close relatives of Spanish patients with familial hypercholesterolemia]. Rev Esp Cardiol 2009;62:57-65

22. Wonderling D, Umans-Eckenhausen MA, Marks D, et al. Costeffectiveness analysis of the genetic screening program for familial hypercholesterolemia in the Netherlands. Semin Vasc Med 2004; 4:97-104.

23. Chen CX, Hay JW. Cost-effectiveness analysis of alternative screening and treatment strategies for heterozygous familial hypercholesterolemia in the United States. Int J Cardiol 2015;181:417-24.

24. Najam O, Ray KK. Familial hypercholesterolemia: a review of the natural history, diagnosis, and management. Cardiol Ther 2015;4:25-38.

25. Sharifi M, Rakhit RD, Humphries SE, et al. Cardiovascular risk stratification in familial hypercholesterolaemia. Heart 2016;102:1003-8.

26. Versmissen J, Oosterveer DM, Yazdanpanah M, et al. Efficacy of statins in familial hypercholesterolaemia: a long term cohort study. BMJ 2008;337:a2423.

27. Civeira F. International panel on management of familial hypercholesterolemia. guidelines for the diagnosis and management of heterozygous familial hypercholesterolemia. Atherosclerosis 2004;173:55-68.

28. Knowles JW, Howard WB, Karayan L, et al. Access to nonstatin lipid-lowering therapies in patients at high risk of atherosclerotic cardiovascular disease. Circulation 2017;135:2204-6.

29. Murray CJ, Lopez AD. Evidence-based health policy--lessons from the global burden of disease study. Science 1996;274:740-3.

30. Austin MA, Hutter CM, Zimmern RL, et al. Familial hypercholesterolemia and coronary heart disease: a HuGE association review. Am J Epidemiol 2004;160:421-9.

31. Hutter CM, Austin MA, Humphries SE. Familial hypercholesterolemia, peripheral arterial disease, and stroke: a HuGE minireview. Am J Epidemiol 2004;160:430-5.

32. Wong B, Kruse G, Kutikova L, et al. Cardiovascular disease risk associated with familial hypercholesterolemia: a systematic review of the literature. Clin Ther 2016;38:1696-709. 
33. Mundal L, Retterstøl K. A systematic review of current studies in patients with familial hypercholesterolemia by use of national familial hypercholesterolemia registries. Curr Opin Lipidol 2016;27:388-97.

34. Henderson R, O'Kane M, McGilligan V, et al. The genetics and screening of familial hypercholesterolaemia. J Biomed Sci 2016;23:39.

35. Stroup DF, Berlin JA, Morton SC, et al. Meta-analysis of observational studies in epidemiology: a proposal for reporting. Meta-analysis Of Observational Studies in Epidemiology (MOOSE) group. JAMA 2000;283:2008-12.

36. Barendregt JJ, Doi SA, Lee YY, et al. Meta-analysis of prevalence. $J$ Epidemiol Community Health 2013;67:974-8.

37. DerSimonian R, Laird N. Meta-analysis in clinical trials. Control Clin Trials 1986;7:177-88.

38. Borenstein M, Hedges LV, Higgins JPT, et al. Identifying and quantifying heterogeneity.In: Introduction to meta-analysis. Hoboken, New Jersey: John Wiley \& Sons, Ltd, 2009:107-25.

39. Egger M, Davey Smith G, Schneider M, et al. Bias in meta-analysis detected by a simple, graphical test. BMJ 1997;315:629-34.

40. Suhail AR, Williams GM, eds. Methods of clinical epidemiology. Berlin, Heidelberg: Springer Berlin Heidelberg, 2013. http://link. springer.com/. (accessed 7 Aug 2016).

41. Duval S, Tweedie R, Trim TR. Trim and fill: a simple funnel-plot-based method of testing and adjusting for publication bias in meta-analysis. Biometrics 2000;56:455-63.

42. Catapano AL, Lautsch D, Tokgözoglu L, et al. Prevalence of potential familial hypercholesteremia $(\mathrm{FH})$ in 54,811 statin-treated patients in clinical practice. Atherosclerosis 2016;252:1-8.

43. de Ferranti SD, Rodday AM, Mendelson MM, et al. Prevalence of familial hypercholesterolemia in the 1999 to 2012 United States National Health and Nutrition Examination Surveys (NHANES). Circulation 2016;133:1067-72.

44. Pajak A, Szafraniec K, Polak M, et al. Prevalence of familial hypercholesterolemia: a meta-analysis of six large, observational, population-based studies in Poland. Arch Med Sci 2016;12:687-96.

45. Watts GF, Shaw JE, Pang J, et al. Prevalence and treatment of familial hypercholesterolaemia in Australian communities. Int $J$ Cardiol 2015;185:69-71.

46. Lahtinen AM, Havulinna AS, Jula A, et al. Prevalence and clinical correlates of familial hypercholesterolemia founder mutations in the general population. Atherosclerosis 2015;238:64-9.

47. Steyn K, Goldberg YP, Kotze MJ, et al. Estimation of the prevalence of familial hypercholesterolaemia in a rural afrikaner community by direct screening for three afrikaner founder low density lipoprotein receptor gene mutations. Hum Genet 1996;98:479-84.

48. Vuorio AF, Turtola H, Piilahti KM, et al. Familial hypercholesterolemia in the finnish north Karelia: a molecular, clinical, and genealogical study. Arterioscler Thromb Vasc Biol 1997;17:3127-38.

49. Perak AM, Ning $\mathrm{H}$, de Ferranti SD, et al. Long-term risk of atherosclerotic cardiovascular disease in US adults with the familial hypercholesterolemia phenotype. Circulation 2016;134:9-19.

50. Yang S, Hwang JS, Park HK, et al. Serum lipid concentrations, prevalence of dyslipidemia, and percentage eligible for pharmacological treatment of Korean children and adolescents; data from the Korea National Health and Nutrition Examination survey IV (2007-2009). PLoS One 2012;7:e49253.

51. Pang J, Martin AC, Mori TA, et al. Prevalence of familial hypercholesterolemia in adolescents: potential value of universal screening? J Pediatr 2016;170:315-6.

52. Benn M, Watts GF, Tybjærg-Hansen A, et al. Mutations causative of familial hypercholesterolaemia: screening of 98098 individuals from the Copenhagen General Population Study estimated a prevalence of 1 in 217. Eur Heart J 2016;37:1384-94.

53. Shi Z, Yuan B, Zhao D, et al. Familial hypercholesterolemia in China: prevalence and evidence of underdetection and undertreatment in a community population. Int J Cardiol 2014;174:834-6.

54. Abul-Husn NS, Manickam K, Jones LK, et al. Genetic identification of familial hypercholesterolemia within a single U.S. health care system. Science 2016;354:aaf7000.
55. Guglielmi V, Bellia A, Pecchioli S, et al. What is the actual epidemiology of familial hypercholesterolemia in Italy? evidence from a national primary care database. Int $J$ Cardiol 2016;223:701-5.

56. Safarova MS, Liu H, Kullo IJ. Rapid identification of familial hypercholesterolemia from electronic health records: the SEARCH study. J Clin Lipidol 2016;10:1230-9.

57. Vickery AW, Ryan J, Pang J, et al. Increasing the detection of FH using general practice electronic databases. Heart Lung Circ 2017;26:450-4.

58. Wald DS, Bestwick JP, Morris JK, et al. Child-parent familial hypercholesterolemia screening in primary care. $N$ Engl $J$ Med 2016;375:1628-37.

59. Sterne JA, Bradburn MJ, Egger M. Meta-analysis in stataTM. In: Systematic reviews in health care: meta-analysis in context. 2nd edn, 2008:347-69.

60. DeSA UNPopulation Division, Department of Economic and Social Affairs. World population prospects: the 2015 revision; 2015.

61. Nordestgaard BG, Chapman MJ, Humphries SE, et al. Familial hypercholesterolaemia is underdiagnosed and undertreated in the general population: guidance for clinicians to prevent coronary heart disease. Eur Heart J 2013;34:3478-90.

62. Henneman L, McBride CM, Cornel MC, et al. Screening for familial hypercholesterolemia in children: what can we learn from Adult Screening Programs? Healthcare 2015;3:1018-30.

63. Hopcroft KA. Child-parent screening may have adverse psychological effects. BMJ 2007;335:683.

64. Calonge N, Guirguis-Blake J. Screening for familial hypercholesterolaemia. BMJ 2007;335:573-4.

65. Wald DS, Bestwick JP, Wald NJ. Child-parent screening for familial hypercholesterolaemia: screening strategy based on a meta-analysis. BMJ 2007;335:599.

66. Weng SF, Kai J, Andrew Neil H, et al. Improving identification of familial hypercholesterolaemia in primary care: derivation and validation of the familial hypercholesterolaemia case ascertainment tool (FAMCAT). Atherosclerosis 2015;238:336-43.

67. WHO, World Health Statistics. Monitoring health for the SDGs. 2016 http://www.who.int/gho/publications/world_health_statistics/2016/ en/ (accessed 9 Sep 2016).

68. Expert Panel on Integrated Guidelines for Cardiovascular Health and Risk Reduction in Children and Adolescents, National Heart, Lung, and Blood Institute. Expert panel on integrated guidelines for cardiovascular health and risk reduction in children and adolescents: summary report. Pediatrics 2011;128:S213-56.

69. Jacobson TA, Maki KC, Orringer CE, et al. National Lipid Association recommendations for patient-centered management of dyslipidemia: part 2. J Clin Lipidol 2015;9:S1-22.e1.

70. Gidding SS, Champagne MA, de Ferranti SD, et al. The agenda for familial hypercholesterolemia: a scientific statement from the American Heart Association. Circulation 2015;132:2167-92.

71. Bibbins-Domingo K, Grossman DC, Curry SJ, et al. Screening for lipid disorders in children and adolescents: US Preventive Services Task Force Recommendation Statement. JAMA 2016;316:625-33.

72. Urbina EM, de Ferranti SD. Lipid screening in children and adolescents. JAMA 2016;316:589-91.

73. Cuchel M, Bruckert E, Ginsberg HN, et al. Homozygous familial hypercholesterolaemia: new insights and guidance for clinicians to improve detection and clinical management. A position paper from the Consensus Panel on Familial Hypercholesterolaemia of the European Atherosclerosis Society. Eur Heart J 2014;35:2146-57.

74. Cochrane handbook for systematic reviews of interventions. http:// handbook.cochrane.org/ (accessed 15 Mar 2016).

75. Armijo-Olivo S, Stiles CR, Hagen NA, et al. Assessment of study quality for systematic reviews: a comparison of the Cochrane Collaboration risk of bias tool and the Effective Public Health Practice Project Quality Assessment Tool: methodological research. J Eval Clin Pract 2012;18:12-18. 